\title{
Comparison of Sound Fields in Regularly-shaped, Long and Flat Enclosures with Diffusely Reflecting Boundaries
}

\author{
Jian Kang ${ }^{\dagger}$ \\ School of Architecture, University of Sheffield, Western Bank, Sheffield, S10 2TN, United Kingdom
}

(Received 19 September 2000; revised 20 April 2002; accepted 13 May 2002)

\begin{abstract}
A radiosity-based simulation model has been developed to study the sound fields in rooms having diffusely reflecting boundaries. Using the model, sound fields in four hypothetical spaces, a cube, a long room and two flat rooms, have been compared. In the cube the spatial distribution of sound pressure level and reverberation time is rather even. In the long rooms and flat rooms, the sound pressure level decreases continuously with increasing source-receiver distance, whilst the reverberation time increases steadily until it reaches a maximum and then decreases slightly. This means that in long and flat rooms the sound fields are fundamentally different from the diffuse field even when the boundaries are diffusely reflective - the reverberation time in these rooms is dependent on the source and receiver location.
\end{abstract}

${ }^{\dagger}$ Member of the International Institute of Acoustics and Vibration (IIAV)

\section{INTRODUCTION}

The inapplicability of classic room acoustical theories in irregularly-shaped spaces such as long ${ }^{1-13}$ and flat rooms ${ }^{14-22}$ has often been reported. It has been demonstrated both theoretically and experimentally that in underground stations (typically long rooms) and large factories (typically flat rooms), the reverberation time (RT) differs significantly from results using Sabine or Eyring formula. The main reason is that in these spaces the sound fields are far from diffuse due to the extreme dimensions of the rooms. Most of the existing studies in this aspect are based on geometrically reflecting boundaries. It would be interesting to investigate the sound fields in such rooms having diffusely reflecting boundaries.

For regularly-shaped rooms, sound fields with diffusely reflecting boundaries have been studied. ${ }^{20-29} \mathrm{~A}$ series of integral formulae has been developed. ${ }^{23-27}$ Since theoretical solutions are not available in most cases, numerical methods must be used. Computation results suggest that for regularly-shaped rooms, although diffuse boundaries do not necessarily produce diffuse fields, the classic Eyring or Sabine formulae can be used as an approximation. For flat rooms, Hodgson has shown that the changes of sound decay and sound propagation that occur with increasing diffuse surface reflection can be very large. ${ }^{20}$ The sound fields in such rooms having diffusely reflecting boundaries are not diffuse and thus classic room acoustical theories are not applicable, especially for the steady-state sound pressure level (SPL).

It would be useful to systematically compare the sound fields between various room shapes, including regularlyshaped, flat and long rooms, particularly with diffusely reflecting boundaries. In this paper, such a comparison is made using a radiosity-based theoretical/computer model. ${ }^{29}$

\section{RADIOSITY MODEL}

The radiosity method, also called radiation balance, radiation exchange or radiant interchange, was first developed in the 19th century for the study of radiant heat transfer in simple configurations. ${ }^{30}$ With the rapid development of computing resources, the techniques have been widely used in computer graphics and lighting simulation. ${ }^{31}$ The use of the radiosity method for analysing acoustics in auditoria was suggested in the $1980 \mathrm{~s}^{25,32,33}$ In a technique developed by Moore ${ }^{32}$, boundaries and receivers are replaced with nodes in a network. The sound propagation is then simulated using the energy exchange between these nodes. Lewers developed a model that integrates Moore's diffusion model and a triangular beam-tracing model. In Lewers' model specular paths are randomised after a certain number of reflections, in order to produce a reasonably diffuse reverberant tail for an impulse response. ${ }^{33}$ In comparison with the application in computer graphics and lighting simulation, an important feature of using the radiosity method in acoustics is that the time factor (reverberation) should be considered and this could bring a significant increase in computation time.

The models developed by Moore and Lewers both implicitly assume a diffuse sound field. Unlike Moore and Lewers, the theoretical/computer model developed in this research divides each boundary into a number of patches and treats every patch as a node. In this way the sound field in a room can be analysed in increased detail, which is essential for non-diffuse fields. In the model the boundaries are diffusely reflective according to the Lambert cosine law, $I(\eta) \sim \cos \eta$, where $\eta$ is the angle between the boundary normal and a reflection. ${ }^{23}$ The simulation procedure in the model is outlined below.

The first step of the model is the division of the boundaries. In the model the boundaries are so divided that a patch is smaller when it is closer to an edge. Using a constant patch size results in the calculation becoming less accurate as it approaches an edge, because in the model the centre-to-centre distance is used to approximate the distance between pairs of patches. For convenience, the patches are all made rectangular, and the patch size is made in the manner of a geometric series. An absorption coefficient can be given for each patch.

International Journal of Acoustics and Vibration, Vol. 7, No. 3, 2002 (pp 165-171) 